\title{
The microbiological characteristics of group B streptococcus at Japanese pediatric hospitals
}

\author{
Mihoko Furuichi' \\ Kaoru Goto ${ }^{2}$ \\ Nobuyuki Tetsuka ${ }^{3}$ \\ Katsushi Ishida ${ }^{4}$ \\ Isao Miyairi ${ }^{3}$ \\ Yoshiaki $\mathrm{Cho}^{5}$ \\ Masashi Kasai ${ }^{6}$ \\ Yukiya Kurahashi ${ }^{7}$ \\ Hideki Akeda ${ }^{7}$ \\ Yuho Horikoshi'
}

'Division of Infectious Diseases, Department of Pediatrics, ${ }^{2}$ Division of Microbiology, Department of Clinical Laboratory, Tokyo Metropolitan Children's Medical Center, ${ }^{3}$ Division of Infectious Diseases, Department of Pediatrics, ${ }^{4}$ Division of Microbiology, Department of Clinical Laboratory, National Center for Child Health and Development, Tokyo, ${ }^{5}$ Department of Pediatrics, ${ }^{6}$ Division of Pediatric Critical Care Medicine and Infectious Disease, Nagano Children's Hospital, Nagano, ${ }^{7}$ Department of General Pediatrics, Okinawa Prefectural Nanbu Medical Center \& Children's Medical Center, Okinawa, Japan

Correspondence: Mihoko Furuichi Division of Infectious Diseases, Department of Pediatrics, Tokyo Metropolitan Children's Medical Center, 2-8-29 Musashidai, Fuchu, Tokyo I83-856I, Japan

$\mathrm{Tel}+8 \mathrm{I} 423005 \mathrm{III}$

Fax +81423128163

Email mihoko.isogai@gmail.com
This article was published in the following Dove Press journal:

Research and Reports in Neonatology

I December 2016

Number of times this article has been viewed

\begin{abstract}
Group B streptococcus (GBS) is an important cause of serious bacterial infections (SBIs) in infants. Non-penicillin-susceptible GBS strains have been reported among adults, but little is known among children. We collected GBS isolates from 4 children's hospitals in Japan, and performed serotyping of GBS strains and reviewed penicillin minimum inhibitory concentrations of GBS strains from both sterile and non-sterile sites. Our examination revealed 235 isolates including 41 isolates from SBIs and 194 isolates from colonization. Nine cases of GBS SBIs (21.9\%) were early-onset, 19 (46.3\%) were late-onset, and 13 (31.7\%) were ultralate-onset. Non-penicillin-susceptible GBS strains were not detected among either isolates. Serotypes Ia, Ib, and III were predominant among SBIs. In conclusion, at this time, we have no non-penicillin-susceptible GBS strains in Japanese children. Penicillin susceptibility should continue to be monitored carefully in each geographic area.
\end{abstract}

Keywords: GBS, serotypes, penicillin, MIC, children

\section{Introduction}

Group B streptococcus (GBS) is one of the major causative pathogens of sepsis and meningitis in infants. In Japan, GBS-related invasive diseases were reported to be 0.13 per 1,000 live births. ${ }^{1}$ In approximately $10-20 \%$ of pregnant women, GBS colonization was detected in the genital tract, where the pathogen is transmitted to infants through delivery. ${ }^{2,3}$ Infants can also acquire GBS by horizontal transmission postnatally.

GBS is categorized into 10 serotypes, some of which predominantly cause serious bacterial infections (SBIs) in infants from colonized serotypes Ia, Ib, III, VI, and V in pregnant women. The frequency rate of common serotypes III, Ia, and Ib in cases of GBS SBIs among Japanese infants was $58.7 \%, 21.3 \%$, and $12.7 \%$, respectively. ${ }^{4}$ Besides those serotypes, serotype II and V were reported in Europe and the US. 5,6 On the other hand, elderly adults have a spectrum of diseases such as pneumonia, meningitis, and sepsis with serotypes Ib, II, and V at 1.3 cases per 1,000 hospitalizations in Japan. ${ }^{7-9}$

GBS is generally susceptible to penicillin, but a low incidence of non-penicillinsusceptible GBS strains has been reported among elderly patients in Japan. ${ }^{10}$ The carriage rate of non-penicillin-susceptible GBS strains among reproductive age women and neonates in Japan is not yet known. Surveillance of susceptibility to penicillin of GBS in each geographic area is important to select intrapartum antibiotic prophylaxis to pregnant women with GBS colonization and empiric therapy to infants with suspected GBS infection. We conducted a retrospective study to identify the rate of 
non-penicillin-susceptible GBS strains among the pediatric population in Japan.

\section{Materials and methods}

In this multicenter study, GBS isolates from sterile and nonsterile specimens were collected from Tokyo Metropolitan Children's Medical Center (Tokyo), National Center for Child Health and Development (Tokyo), Nagano Children's Hospital (Nagano), and Nanbu Child Medical Center (Okinawa) in Japan between January, 2008 and January, 2014. Serotypes of GBS from SBIs strains were typed by commercial GBS serotyping kit, which uses serotype-specific rabbit immunoglobulin (Denka Seiken, Tokyo, Japan). The minimum inhibitory concentration (MIC) was measured by broth microdilution method. The European Committee on Antimicrobial Susceptibility Testing (EUCAST) breakpoint was used. Penicillin MICs $\leqq 0.12$ and $>0.12$ were interpreted as susceptible and non-susceptible, respectively. Clinical charts were reviewed for age, sex, and type of invasive disease. Early-onset, late-onset, and ultra-late-onset diseases were defined according to timing of disease onset in the first week, 1 week to 3 months, and over 3 months, respectively. Bacteremia and meningitis were confirmed by a positive blood or cerebrospinal fluid culture. Cellulitis was diagnosed on the basis of skin and soft tissue infection with a positive blood culture. Necrotizing fasciitis was diagnosed on the basis of skin and soft tissue infection with a positive tissue culture from surgical debridement. Pneumonia was diagnosed on the basis of respiratory symptoms, positive $\mathrm{X}$-ray findings, and positive sputum culture from the tracheal tube. This study was exempt from obtaining ethical approval and written informed consent as per the Japanese National Guideline for observational studies conducted before 2014.

\section{Results}

We identified 235 GBS strains. The mean age was day 64 (SBIs: day 38, colonization: day 64.5). The specimens were collected from 109 girls and 126 boys. Forty-one (17.4\%) isolates were derived from SBIs and 194 (82.6\%) isolates were derived from colonization. Nine cases of GBS SBIs $(21.9 \%)$ were early-onset, $19(46.3 \%)$ were late-onset, and 13 $(31.7 \%)$ were ultra-late-onset. The invasive diseases, bacteremia, meningitis, pneumonia, cellulitis, urinary tract infection, and necrotizing fasciitis were detected at a frequency of 22 (55\%), 10 (24.3\%), 5 (12.1\%), 2 (4.8\%), 1 (3.8\%), and 1 $(3.8 \%)$, respectively.

Among the 41 isolates from SBIs, 37 were serotyped. The Ia, Ib, II, III, V, and VI serotypes of GBS occurred at a

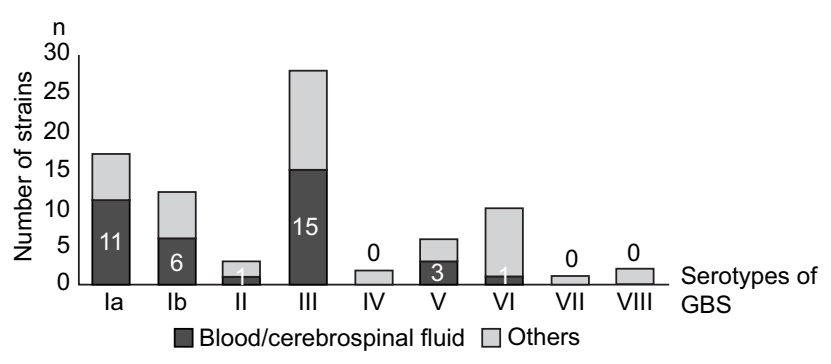

Figure I Serotypes of group B streptococcus (GBS) isolated from blood or cerebrospinal fluid and others.

Notes: Others refer to skin, sputum, urine, and stool. These were not sterile specimens.

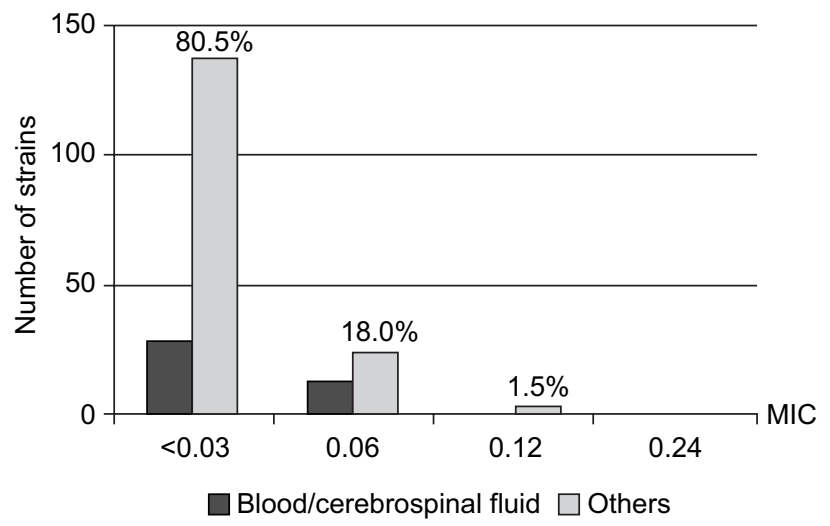

Figure 2 Penicillin minimum inhibitory concentration of 205 group B streptococcus strains isolated from blood or cerebrospinal fluid and others.

Notes: Others refer to skin, sputum, urine, and stool. These were not sterile specimens.

Abbreviation: MIC, minimum inhibitory concentration.

frequency of $11(29.8 \%), 6(16.2 \%), 1(2.7 \%), 15(40.5 \%)$, $3(8.1 \%)$, and $1(2.7 \%)$, respectively (Figure 1$)$.

The penicillin MICs were determined for $90.2 \%$ $(212 / 235)$ of the isolates, none of which were found to be resistant to penicillin. The penicillin MIC was $<0.03 \mu \mathrm{g} / \mathrm{ml}$ for $77.8 \%$ (165/212) of the isolates, $0.06 \mu \mathrm{g} / \mathrm{ml}$ for $17.4 \%$ $(37 / 212)$ of the isolates, and $0.12 \mu \mathrm{g} / \mathrm{ml}$ for $1.4 \%(3 / 212)$ of the isolates. Non penicillin-susceptible strains were not detected. All 3 isolates for which the MIC was $0.12 \mu \mathrm{g} / \mathrm{ml}$ were derived from colonization (Figure 2).

\section{Discussion}

This study was conducted to survey GBS penicillin susceptibility among children in Japan. We did not identify any non-penicillin-susceptible strains in the cases of either SBI or colonization. However, $1.4 \%$ (3/212) of strains from colonization had an MIC of $0.12 \mu \mathrm{g} / \mathrm{ml}$, which was the upper limit of the susceptibility range. We may still administer penicillin to mothers for GBS prophylaxis and SBIs treatment in infants, but penicillin susceptibility should be monitored carefully for emergence of non-susceptible strains. 
Unlike Streptococcus pyogenes, non-penicillin-susceptible GBS strains of Ib, II, and V were reported among elderly patients in Japan. ${ }^{10}$ Serotype $\mathrm{Ib}$ is one of the strains that causes invasive diseases in infants. The isolated serotype $\mathrm{Ib}$ in our study was fully penicillin susceptible. Decreased susceptibility to beta-lactam antibiotics in GBS was related with $p b p 2 x$ mutation. ${ }^{11}$ Further monitoring on susceptibility of GBS is needed.

After introduction of practice guidelines to prevent vertical transmission of GBS, early-onset GBS infections in neonates were decreased from 0.47 per 1,000 live births in 1999-2001 to 0.34 per 1,000 live births in 2003-2005 in the US. ${ }^{6}$ On the other hand, the rate of early-onset GBS infections in the Netherlands was increased from 0.11 per 1,000 live births in 1987 to 0.19 per 1000 live births in 2011, although they implemented similar practice guidelines to prevent vertical transmission. ${ }^{12}$ In Japan, the rates of early-onset GBS infections did not change before and after implementation of prevention guidelines at delivery in 2008 , which were 0.08 per 1,000 live births in 2004-2008 and 0.10 per 1,000 live births in 2009-2010, respectively. ${ }^{13,14}$ The Netherlands and Japan had much lower incidence of GBS diseases than the US, so those countries with low incidence of early-onset GBS diseases might not have benefited from prevention strategy.

The rate of late-onset GBS infections increased to 0.34 per 1,000 live births during 2003-2005 in the US. ${ }^{6}$ The rate of late-onset GBS diseases in Japan remained at 0.10 per 1,000 live births through 2004-2010, which was comparable to early-onset GBS diseases. ${ }^{13}$ Our study found more lateonset GBS diseases (46.3\%) than early-onset GBS diseases $(21.9 \%)$. This may be explained by sampling bias as the study was conducted at only tertiary children's hospitals, which tend to admit neonates who need surgery rather than preterm or infants with severe illness who were discharged home after birth. Early-onset GBS infection might be taken care of at local general hospitals with neonatal intensive care units in Japan.

GBS polysaccharide-protein conjugate vaccine, under development for serotypes Ia, Ib, and III, was found to induce an immune response in pregnant women and sustain a protective level of antibody in their infants up to 2 months of age. A trivalent GBS vaccine may be a reasonable choice for introduction into Japan based on this epidemiology. ${ }^{15}$ Surveillance of serotypes of GBS is important in terms of public health to understand invasive GBS diseases. Particularly, recent advances in GBS vaccine may benefit infants for preventing both early- and late-onset diseases.
Our study had some limitations. First, sample size was small to generalize our finding. Second, the rate of early-onset GBS disease was only $22 \%$. Potential different characteristics between early-onset and late-onset strains might exist because serotypes were likely to be different between them. In this study, since the rate of early-onset GBS disease was only $22 \%$, the results of this study should be carefully interpreted when applying to the early-onset sepsis. More GBS isolates are needed to consider non-susceptible strains in early-onset disease.

In conclusion, no non-penicillin-susceptible strain was detected. We should monitor GBS susceptibility to penicillin continuously.

\section{Acknowledgment}

The authors thank Rumi Okuno, Tokyo Metropolitan Institute of Public Health, for performing GBS serotyping. This study was presented at the 32nd annual meeting of the European Society for Paediatric Infectious Diseases in Dublin, Ireland, during 6-10 May, 2014.

\section{Disclosure}

The authors report no conflicts of interest in this work.

\section{References}

1. Chang B, Wada A, Hosoya M, et al. Characteristics of group B Streptococcus isolated from infants with invasive infections: a population-based study in Japan. Jpn J Infect Dis. 2014;67(5):356-360.

2. Romanik M, Nowosielski K, Poreba R, Sioma-Markowska U, Martiroisian G, Groborz J. Streptococcus group B serotype distribution in anovaginal isolates of women in term pregnancy. Neuro Endocrinol Lett. 2014;35(4):301-305.

3. Itakura A, Kurauchi O, Morikawa S, Matsuzawa K, Mizutani S, Tomoda Y.A prospective study on the relationship between intrapartum maternal group-B streptococcal concentration and signs of infection in neonates. J Obstet Gynaecol Res. 1996;22(2):101-105.

4. Morozumi M, Wajima T, Kuwata Y, et al. Associations between capsular serotype, multilocus sequence type, and macrolide resistance in Streptococcus agalactiae isolates from Japanese infants with invasive infections. Epidemiol Infect. 2014;142(4):812-819.

5. Lamagni TL, Keshishian C, Efstratiou A, et al. Emerging trends in the epidemiology of invasive group B streptococcal disease in England and Wales, 1991-2010. Clin Infect Dis. 2013;57(5):682-688.

6. Phares CR, Lynfield R, Farley MM, et al; Active Bacterial Core surveillance/Emerging Infections Program Network. Epidemiology of invasive group B streptococcal disease in the United States, 1999-2005. JAMA. 2008;299(17):2056-2065.

7. Murayama SY, Seki C, Sakata H, et al; Invasive Streptococcal Disease Working Group. Capsular type and antibiotic resistance in Streptococcus agalactiae isolates from patients, ranging from newborns to the elderly, with invasive infections. Antimicrob Agents Chemother. 2009;53(6):2650-2653.

8. Ueno H, Yamamoto Y, Yamamichi A, Kikuchi K, Kobori S, Miyazaki M. Characterization of group B streptococcus isolated from women in Saitama city, Japan. Jpn J Infect Dis. 2012;65(6):516-521.

9. Matsubara K, Yamamoto G. Invasive group B streptococcal infections in a tertiary care hospital between 1998 and 2007 in Japan. Int J Infect Dis. 2009;13(6):679-684. 
10. Kimura K, Nagano N, Nagano Y, et al. Predominance of sequence type 1 group with serotype VI among group B streptococci with reduced penicillin susceptibility identified in Japan. JAntimicrob Chemother. 2011; 66(11):2460-2464.

11. Dahesh S, Hensler ME, Van Sorge NM, et al. Point mutation in the group B streptococcal pbp2x gene conferring decreased susceptibility to beta-lactam antibiotics. Antimicrob Agents Chemother. 2008; 52(8):2915-2918

12. Bekker V, Bijlsma MW, van de Beek D, Kuijpers TW, van der Ende A. Incidence of invasive group B streptococcal disease and pathogen genotype distribution in newborn babies in the Netherlands over 25 years: a nationwide surveillance study. Lancet Infect Dis. 2014;14(11):1083-1089.
13. Matsubara K, Hoshina K, Suzuki Y. Early-onset and late-onset group B streptococcal disease in Japan: a nationwide surveillance study, 2004-2010. Int J Infect Dis. 2013;17(6):e379-e384.

14. Minakami H, Maeda T, Fujii T, et al. Guidelines for obstetrical practice in Japan: Japan Society of Obstetrics and Gynecology (JSOG) and Japan Association of Obstetricians and Gynecologists (JAOG) 2014 edition. J Obstet Gynaecol Res. 2014;40(6):1469-1499.

15. Madhi SA, Dangor Z, Heath PT, et al. Considerations for a phaseIII trial to evaluate a group B Streptococcus polysaccharide-protein conjugate vaccine in pregnant women for the prevention of early- and late-onset invasive disease in young-infants. Vaccine. 2013;31 Suppl 4: D52-D57.

\section{Publish your work in this journal}

Research and Reports in Neonatology is an international, peer-reviewed, open access journal publishing original research, reports, editorials, reviews and commentaries on neonatal health. The manuscript management system is completely online and includes a very quick and fair peer-review system. Visit http://www.dovepress.com/testimonials.php to read real quotes from published authors. 